\title{
Differences in Molecular Characteristics of Segment 8 in Rice black-streaked dwarf virus and Southern rice black-streaked dwarf virus
}

Yu Zhou, Xiaoming Zhang, and Dandan Wang, Northeast Agricultural University, Xiangfang District, Harbin, Heilongjiang Province, China; Jianfeng Weng, Institute of Crop Science, Chinese Academy of Agricultural Sciences, Haidian District, Beijing, China; Hong Di, Lin Zhang, Ling Dong, Hong Zhang, and Hongyue Zu, Northeast Agricultural University, Harbin, China; Xinhai Li, ${ }^{\dagger}$ Institute of Crop Science, Chinese Academy of Agricultural Sciences, Haidian District, Beijing, China; and Zhenhua Wang, ${ }^{\dagger}$ Northeast Agricultural University, Harbin, China

\begin{abstract}
Rice black-streaked dwarf virus (RBSDV) and Southern rice blackstreaked dwarf virus (SRBSDV) cause maize rough dwarf disease (MRDD) and rice black-streaked dwarf disease (RBSDD) in China. RBSDV segment 8 (S8) contains the only deletion mutation in the genomes of these viruses, which are both members of the genus Fijivirus. To illuminate the molecular differences between the RBSDV and SRBSDV genomes and better understand the evolution of these viruses, and to determine which virus is specifically associated with MRDD and RBSDD in each region, $\mathrm{S} 8$ was analyzed in 66 virus isolates collected from 10 geographic locations in China and 14 S8 sequences obtained from the National Center for Biotechnology Information GenBank. Phylogenetic analysis showed that the pathogen associated with MRDD and RBSDD in the Yellow and Huai River valleys was RBSDV, whereas the pathogen associated with these diseases in Sanya was SRBSDV. Codon usage bias in S8 differed significantly between RBSDV and SRBSDV, as indicated by effective number of codons used by a gene $(N c)$ and GC values, $N c$ plots, and variation explained by the first axis in correspondence analysis. The nucleotide identities among these 66 RBSDV and SRBSDV isolates ranged from 66.2 to $68.2 \%$, and were considerably lower than the nucleotide

identities within RBSDV (from 94.1 to $99.9 \%$ ) or SRBSDV (from 93.9 to $100 \%$ ) isolates. Most S8 polymorphisms were identified in the region from 1,000 to $1,200 \mathrm{bp}$ in RBSDV and in the region from 500 to $700 \mathrm{bp}$ in SRBSDV. The difference in the lengths of RBSDV (1,936 bp) and SRBSDV $(1,928 \mathrm{bp})$ was due to an 8 -bp deletion in the $3^{\prime}$-untranslated region of SRBSDV. Six recombination events were detected in S8 in RBSDV and two recombination events were detected in S8 in SRBSDV. Recombination breakpoints were found within the region containing the deletion mutation in nine isolates. However, no recombination events were detected between RBSDV and SRBSDV. Both of these viruses were under negative and purifying selection, although the ratio of nonsynonymous mutations to synonymous mutations $(K a / K s)$ for RBSDV S8 (0.0530) was not significantly lower than that of SRBSDV S8 $(0.0823, P=0.1550)$. We found that SRBSDV was more highly genetically differentiated (product of effective population size and the migration rate among populations $<1$; values for the among-populations component of genetic variation or normalized variation $>0.33$; and $P$ values of the sequence statistic, the rank statistic, and the nearest-neighbor statistic $<0.01)$ than RBSDV. However, gene flow between RBSDV and SRBSDV was not frequent.
\end{abstract}

Maize rough dwarf disease (MRDD) is a destructive viral disease that occurs in maize-growing regions around the world. In China, this disease has mainly been caused by Rice black-streaked dwarf virus (RBSDV) (Li et al. 2012; Yin et al. 2013) and Southern rice black streaked dwarf virus (SRBSDV) (Hoang et al. 2011). In 2008, onefourth of the maize-producing area in Shandong Province was affected, with disease incidences ranging from 30 to $50 \%$, or higher than $80 \%$ in areas with severe epiphytotics (Yin et al. 2011). SRBSDV was first found in China in Guangdong Province (Zhou et al. 2008) but is now found in many provinces in southern China and has caused serious maize losses (Cheng et al. 2013; Wang et al. 2010).

The closely related viruses RBSDV and SRBSDV belong to the genus Fijivirus and exhibit similar disease symptoms, host ranges,

${ }^{\dagger}$ Corresponding authors: Z. Wang; E-mail: zhenhuawang_2006@163.com; and X. Li; E-mail: lixinhai@caas.cn

Y. Zhou and X. Zhang contributed equally to this research.

Funding: This study was funded by the China Postdoctoral Science Foundation Grant (2017M611344), the Young Talents Project of Northeast Agricultural University (17QC03), Heilongjiang Postdoctoral Financial Assistance (LBH-Z16019), University Nursing Program for Young Scholars with Creative Talents in Heilongjiang Province (UNPYSCT-2017011), the Agricultural Science and Technology Innovation Program at CAAS, and the National Key Research and Development Program of China (grant number 2016YFD0101201).

*The $\boldsymbol{e}$-Xtra logo stands for "electronic extra" and indicates that two supplementary tables are published online.

Accepted for publication 27 December 2017.

(c) 2018 The American Phytopathological Society and serology. Both of these viruses are 75-nm icosahedral particles (Shikata and Kitagawa 1977; Zhou et al. 2008). Monoclonal antibodies against SRBSDV and RBSDV cannot distinguish these two viruses because no epitopes differ between them (Liu et al. 2013). The small brown planthopper (SBPH; Laodelphax striatellus Fallén) vectors RBSDV, which is prevalent in most of eastern and northern China (Cheng et al. 2013; Zhou et al. 2011). However, the white-backed planthopper (WBPH; Sogatella furcifera Horváth) transmits SRBSDV, which is prevalent mainly in southern China (Zhou et al. 2013). The nucleotide and amino acid sequences of SRBSDV and RBSDV are highly similar, with identity values ranging from 60 to $80 \%$, and cannot be distinguished by agarose or polyacrylamide gel electrophoresis (Wang et al. 2003; Zhang et al. 2001a). RBSDV and SRBSDV sequences can only be distinguished by sequence alignment.

The genomes of RBSDV and SRBSDV are each composed of 10 double-stranded RNA that have been designated S1 through S10 according to their electrophoretic migration in agarose gels, from slow to fast. The 10 RBSDV segments range in length from 1.8 to $4.5 \mathrm{~kb}$ (Hoang et al. 2011; Marzachí et al. 1991) but those of SRBSDV range from approximately $1.4 \mathrm{~kb}$ to $4.5 \mathrm{~kb}$ (Wang et al. 2012; Yin et al. 2011; Zhang et al. 2008). Most genomic segments of these viruses contain only one open reading frame (ORF); however, S5, S7, and S9 each contain two ORF (Yin et al. 2011, 2013). The nucleotide identities of RBSDV S9 and S10 are $>90.0 \%$, and the amino acid identities of ORF8 and ORF10 are $>96.3 \%$ (Yin et al. 2013). The nucleotide sequence identities of S10 among 15 SRBSDV isolates were $>97.7 \%$ (Cheng et al. 2013). However, the nucleotide identities of S7, S8, S9, and S10 between SRBSDV and RBSDV were all less than 78.4\% (Yin et al. 2011). The sequences identities between RBSDV and SRBSDV were much lower than within RBSDV or SRBSDV populations (Zhou et al. 2008). A single 9-bp deletion mutation was detected in RBSDV S8; the length of S8 in some RBSDV isolates was $1,927 \mathrm{bp}$, while that in others was 1,936 bp. This deletion was in the $3^{\prime}$-untranslated region (UTR); thus, it 
did not affect the amino acid sequence of the protein encoded by S8 (Zhou et al. 2017). No insertion or deletion mutations were found in SRBSDV.

RBSDV S8 encodes a $68-\mathrm{kDa}$ minor core capsid protein (P8) comprising 591 amino acids (Isogai et al. 1998). P8 forms homodimers in insect cells and plants in vitro and functions as a potent transcriptional repressor (Liu et al. 2007). SRBSDV S8 is similar to RBSDV S8 but encodes a major core structural protein (Zhang et al. 2001b). The molecular characteristics of RBSDV and SRBSDV have not previously been analyzed, particularly for S8, which includes the only deletion mutation in the RBSDV genome. In the present study, 66 isolates from maize and rice collected in different years and locations and 14 known S8 sequences retrieved from National Center for Biotechnology Information were used to analyze S8 and gain insights into the phylogeny, diversity, and genetic structure of RBSDV and SRBSDV, and to determine which virus is associated with MRDD and Rice black-streaked dwarf disease (RBSDD) in each region.

\section{Materials and Methods}

Collection of virus isolates. Maize and rice plants that exhibited symptoms of rough dwarf disease were collected from 2012 through 2015 from 10 geographical locations (I through X) in China (Fig. 1) that have typically been affected by the pathogens RBSDV and SRBSDV. In total, 50 maize and 16 rice plants were collected. These included 6 maize plants that were collected from location I, 5 from location II, 5 from location III, 4 from location IV, 5 from location V, 5 from location VI, 6 from location VII, 2 from location VIII, 1 from location IX, and 11 from location X. Two rice plants were collected from location III, five from location V, four from location VI, and five from location VIII (Fig. 1). Plant samples were then stored at $-80^{\circ} \mathrm{C}$. The 14 sequences obtained from the GenBank database include 3 RBSDV S8 sequences and 11 SRBSDV S8 sequences. The GenBank accession numbers of the 66 sequences analyzed in the present study and 14 known sequences from GenBank are shown in Supplementary Table S1.

RNA extractions, reverse-transcription polymerase chain reaction, and sequencing. Total RNA was extracted from infected maize or rice leaves according to previously published methods (Morris and Dodds 1979; Wang et al. 2003), and the quality, integrity, and concentrations of RNA were assessed on $1.5 \%$ native agarose gels and using a NanoDrop 2000 spectrophotometer (Thermo Scientific). We used a Fast Quant RT Kit (TransGen Biotech) to synthesize first-strand cDNA from virus RNA, and then polymerase chain reaction (PCR) amplification of S8 was carried out using KOD-Plus-Neo enzyme (TOYOBO) and primers shown in Supplementary Table S2. The dideoxy chain-termination method was used to sequence the S8 amplification products at the AuGCT DNA-SYN Biotechnology Company. To confirm sequence quality, three independent PCR were sequenced for partial S8 sequences. DNAMAN and Jemboss $1.5 \mathrm{soft}-$ ware were used to assemble and analyze these sequences (EMBOSS) (Carver and Mullan 2005).

Phylogenetic and recombination analysis. Sequences were checked visually and aligned using ClustalW, and a phylogenetic tree was constructed using the neighbor-joining method in MEGA 6.06 software (Tamura et al. 2013) with 1,000 bootstrap replicates. Only bootstrap values $>50 \%$ are shown.

Seven algorithms in RDP 4.22 software (Martin et al. 2010), including RDP, BOOTSCAN, GENECONV, CHIMAERA, Maximum Chi Square, 3Seq, and Sister Scanning, were used to detect possible recombination sites in RBSDV and SRBSDV S8 sequences. Those that were detected by at least three of these methods were considered confirmed. A $P$ value cutoff of 0.05 was adopted and the default number (100) of simulated datasets was used (Martin et al. 2010).

Analysis of codon usage bias in S8 from RBSDV and SRBSDV. CodonW 1.4.4 (https://sourceforge.net/projects/codonw/) was used to assess codon usage in P8 encoded by $\mathrm{S} 8$ from RBSDV and SRBSDV. Several parameters related to codon usage bias, such as $\mathrm{A} 3_{\mathrm{S}}, \mathrm{U} 3_{\mathrm{S}}, \mathrm{C} 3_{\mathrm{S}}$, $\mathrm{G}_{\mathrm{S}}$, and $\mathrm{GC}_{\mathrm{S}}$ (representing the frequencies of $\mathrm{A}, \mathrm{U}, \mathrm{C}, \mathrm{G}$, and $\mathrm{G}+\mathrm{C}$, respectively, appearing in the third amino acid position in codons), the effective number of codons used by a gene $(N c)$, the codon adaptation index $(C A I)$, the codon bias index $(C B I)$, and relative synonymous codon usage ( $R S C U$ ) were calculated for ORF8 from the $66 \mathrm{~S} 8$ sequences obtained from the collected samples (Sau et al. 2006; Sharp and Li 1987; Wright 1990; Zhou et al. 2017).

Analysis of nucleotide diversity and sequence variants. The S8 nucleotide sequences from RBSDV and SRBSDV were aligned using MEGA 6.06 (Tamura et al. 2013). The nucleotide diversity $(\pi)$ of S8 sequences from isolates collected from 10 geographic locations, from maize or rice hosts, and from different years was calculated using TASSEL 3.0 software, with 200-bp sliding windows in 100-bp steps (Weng et al. 2013). Sequence identity was compared using MegAlign in DNAStar 5.01 software. DnaSP 5.0 was used to analyze the number

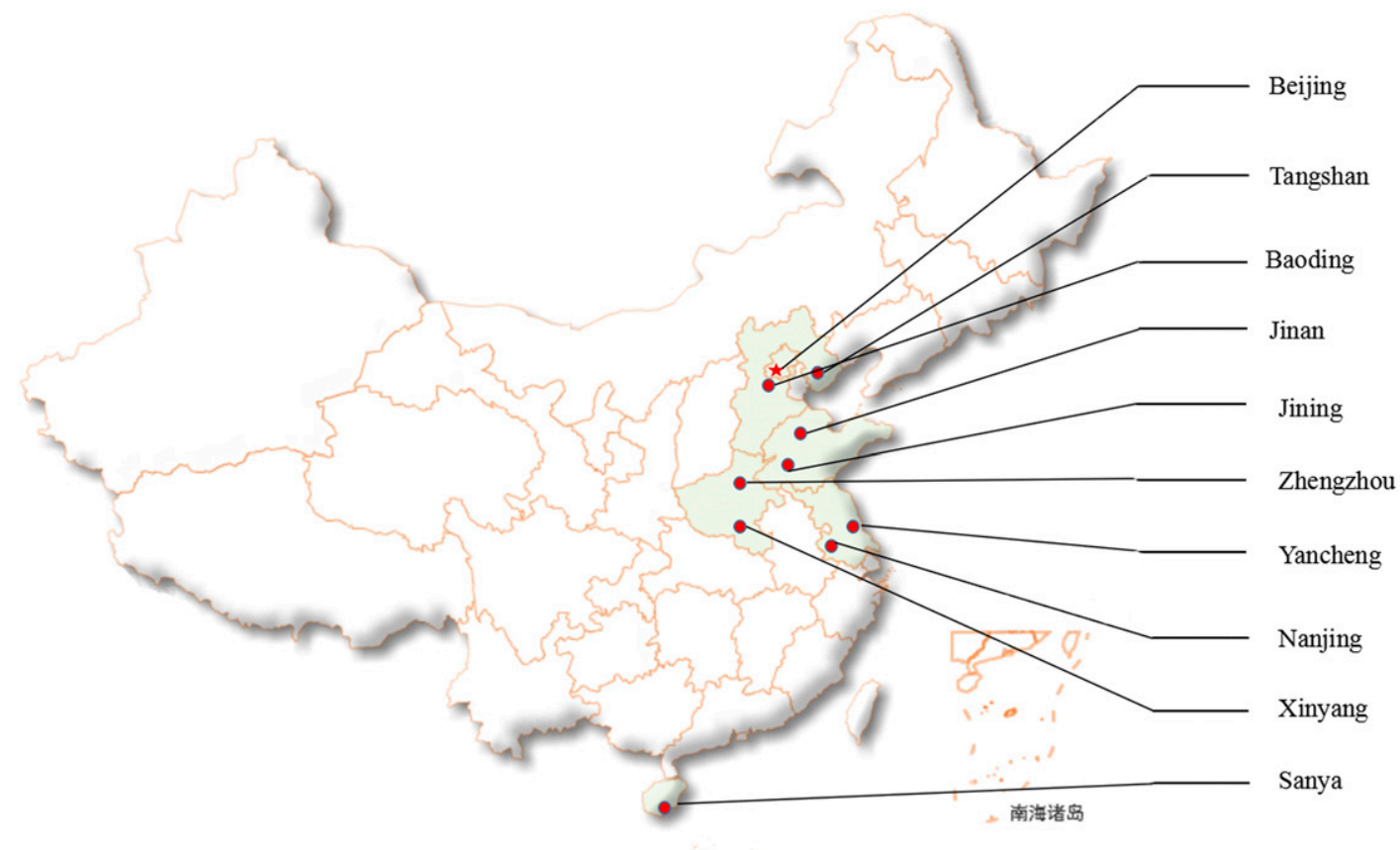

Fig. 1. Geographic distribution of diseased plants collected from the following regions in China: I = Beijing; II = Tangshan, Hebei Province; III = Baoding, Hebei Province; IV = Jinan, Shandong Province; V = Jining, Shandong Province; VI = Zhengzhou, Henan Province; VII = Yancheng, Jiangsu Province; VIII = Nanjing, Jiangsu Province; IX = Xinyang Henan Province; and $\mathrm{X}=$ Sanya, Hainan Province. 
and type of nucleotide variants and to calculate $\pi$ and haplotype diversity (Librado and Rozas 2009). The $\pi$ measures the degree of molecular polymorphism within a population, whereas haplotype diversity indicates how unique a haplotype is within a population.

Analysis of selection pressure on S8 in RBSDV and SRBSDV. Nonsynonymous mutation $(K a)$ and synonymous mutation $(K s)$ values and the $K a / K s$ ratio of S8 from RBSDV and SRBSDV were calculated separately, according to host, location, and year (Pamilo and Bianchi 1993; Tamura et al. 2013).

Selective neutrality was tested by calculating Tajima's D statistic to compare the proportion of polymorphic sites to $\pi$ (Librado and Rozas 2009; Tajima 1989). Fu and Li's D was calculated to assess the differences between the number of singletons and the total number of mutations, and $\mathrm{Fu}$ and $\mathrm{Li}$ 's $\mathrm{F}$ was calculated to assess the

A

A B

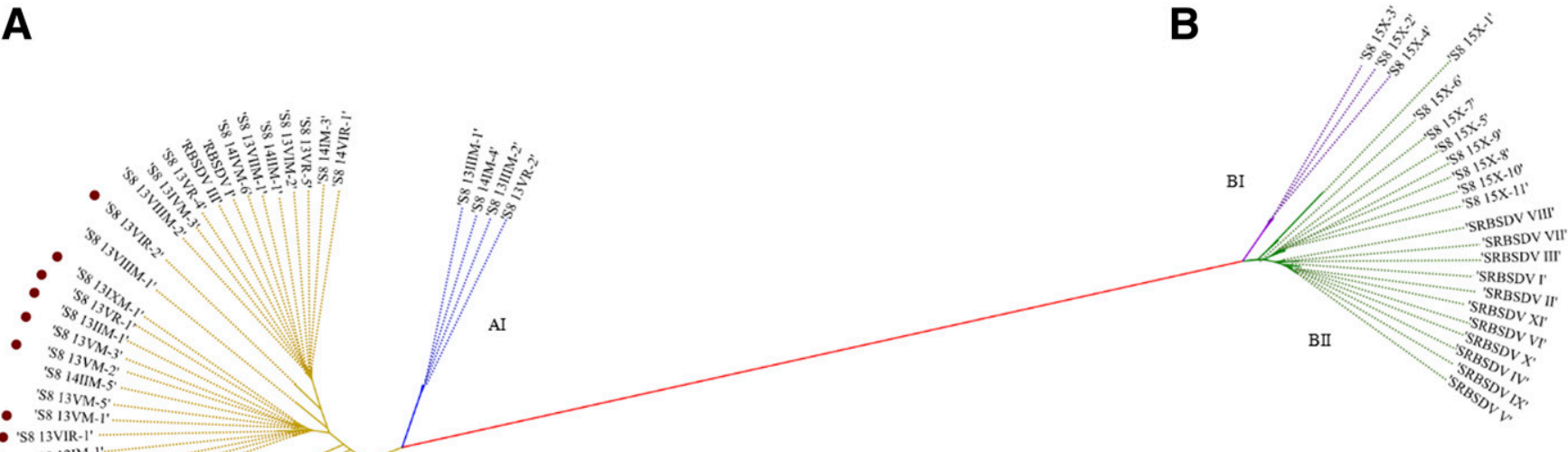

- $\quad \mathrm{s} 812 \mathrm{M} \mathrm{M}-1$

- is8 invilir-4i

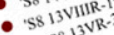

$.288^{2}$

- 2 sitis

. $58.1+4.4-5$

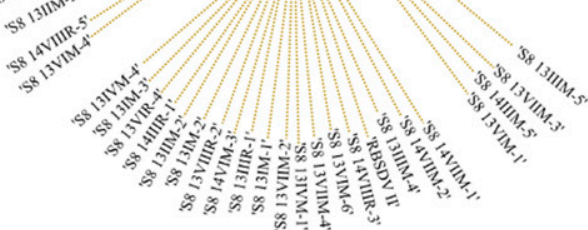

Tree scale: 0.1

Fig. 2. Phylogenetic tree of Rice black-streaked dwarf virus (RBSDV) (A) and Southern rice black-streaked dwarf virus (SRBSDV) (B) segment 8 sequences from collected isolates.
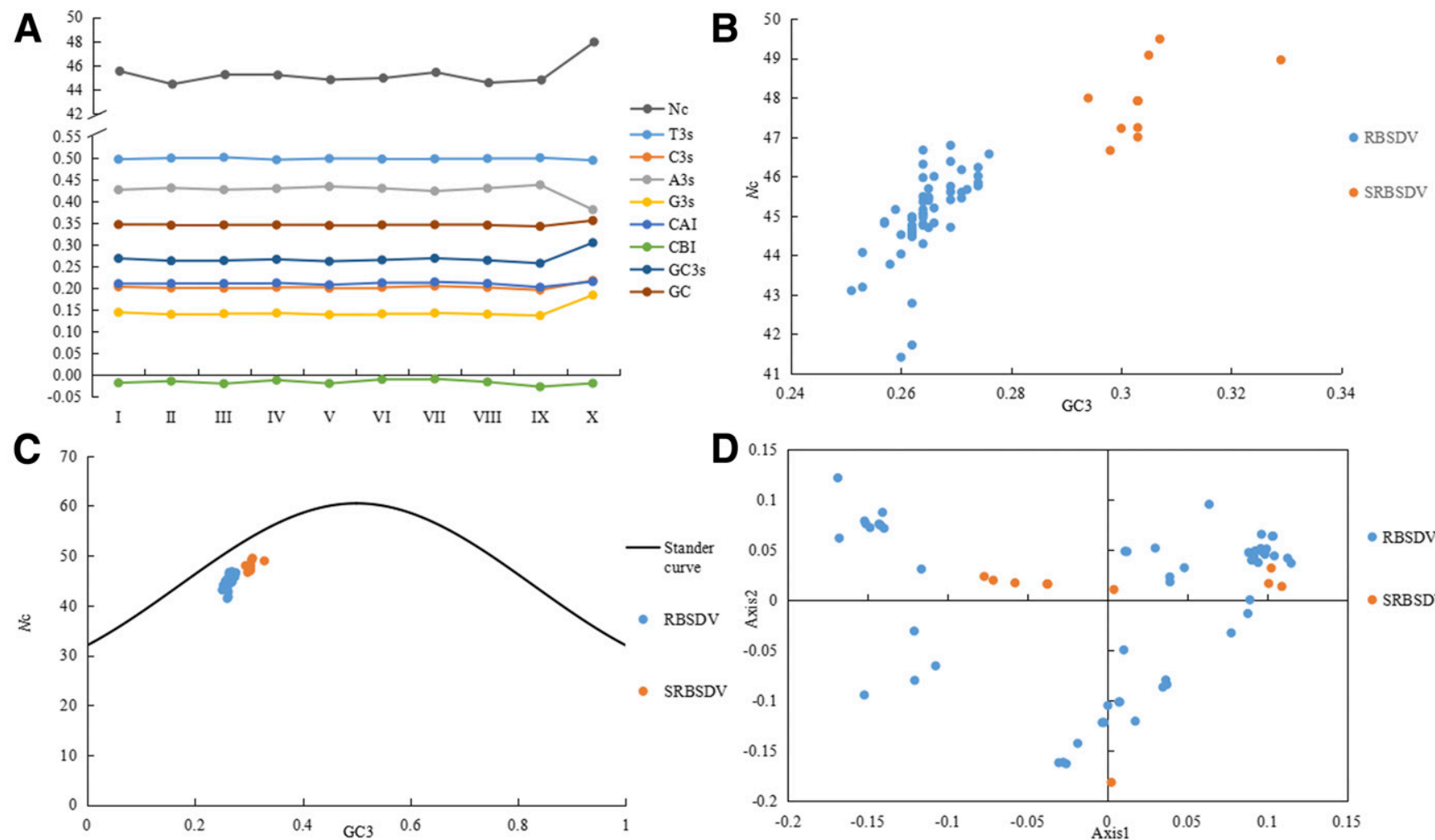

Fig. 3. Codon usage characteristics in Rice black-streaked dwarf virus (RBSDV) segment 8 (S8) and Southern rice black-streaked dwarf virus (SRBSDV) S8. A, effective number of codons used by a gene $(\mathrm{Nc}), \mathrm{T} 3_{\mathrm{s}}, \mathrm{C} 3_{\mathrm{s}}, \mathrm{A} 3_{\mathrm{s}}, \mathrm{G} 3_{\mathrm{s}}$, codon adaptation index (CAl), the codon bias index (CBI), GC3s, and $\mathrm{GC}$ values of isolates from different geographic locations. B, Distribution of $\mathrm{Nc}$ and $\mathrm{GC}_{\mathrm{S}}$ in isolates from 10 geographic locations. C, Distribution of $\mathrm{Nc}$ and $\mathrm{GC} 3_{\mathrm{S}}$ in $\mathrm{S} 8$ sequences. The solid line (shown in black) indicates the standard $\mathrm{Nc}$ value if codon bias is due only to $\mathrm{GC}_{\mathrm{S}}$. $\mathbf{D}$, Correspondence analysis of relative synonymous codon usage in S8 along the first and second axis.

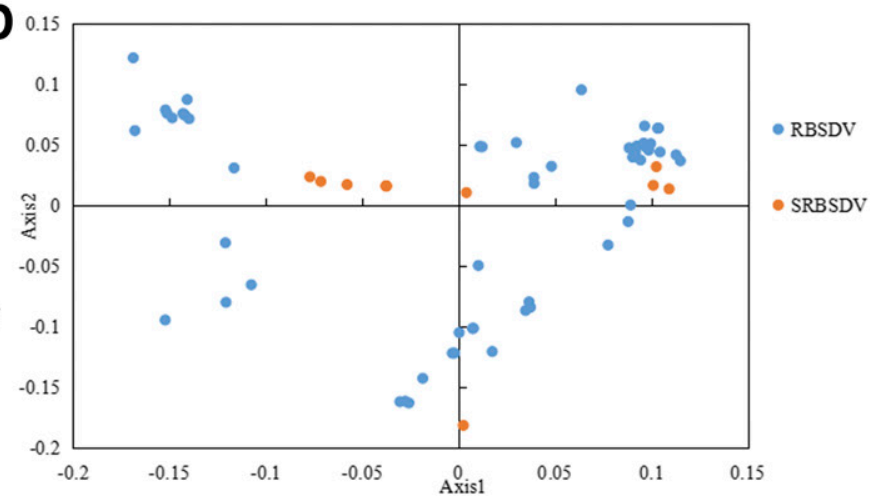


differences between the number of singletons and the average number of nucleotide differences between paired sequences of average nucleotide length (Shackelton et al. 2006). The preceding parameters and haplotype diversity were estimated using DnaSP 5.0 software (Rozas 2009).

Evaluation of genetic differentiation and gene flow. Genetic differentiation parameters in different subpopulations were computed as the sequence statistic $\left(K s^{*}\right)$, the rank statistic $(Z)$, and the nearest-neighbor statistic (Snn). These statistics are more powerful when mutation rates are high and sample sizes are small (Hudson 2000; Hudson et al. 1992). Values for the among-populations component of genetic variation or normalized variation $(F s t)$ and the product of effective population size and the migration rate among populations $(\mathrm{Nm})$ (Rozas 2009) were calculated to estimate the level of gene flow. Infrequent gene flow is assumed to have occurred if the absolute value of $F s t>0.33$. Fst ranges from zero to one in undifferentiated to fully differentiated populations. If $\mathrm{Nm}<1$, considerable local differentiation can result in genetic drift, but if $N m>1$, drift becomes less likely (Wright 1951). DnaSP 5.10 software was used to calculate statistics and estimate genetic differentiation (Librado and Rozas 2009).

\section{Results}

Phylogenetic analysis of S8 from RBSDV and SRBSDV. To determine the evolutionary relationships among these 80 RBSDV and SRBSDV isolates and their classification, a phylogenetic tree was constructed using the $66 \mathrm{~S} 8$ sequences obtained during this research and the $14 \mathrm{~S} 8$ sequences available from GenBank. These 80 isolates could be classified into two main groups (A or B) based on S8 sequences, regardless of host geographic origin. Group A contained 58 isolates, including 6 from location I, 5 from II, 9 from III, 4 from IV, 11 from V, 9 from VI, 6 from VII, 7 from VIII, and 1 from IX, in addition to $3 \mathrm{~S} 8$ sequences from GenBank that were annotated as
RBSDV. Group B contained 11 isolates from $X$ and 11 sequences from GenBank that were annotated as SRBSDV. Both groups A and B could be further divided into two subgroups each. Group A1 contained four isolates, including two from location III in 2013, one from V in 2013, and one from I in 2014. Group A2 contained 52 isolates and 3 RBSDV sequences from GenBank. Group B1 contained three isolates from location X. Group B2 contained 8 isolates from location $\mathrm{X}$ and 11 SRBSDV sequences from GenBank. The 9-bp deletion mutation was detected in 13 isolates from group AII that were closely related (Fig. 2). According to these results, the isolates from the Yellow and Huai River valleys (from locations I through IX) were all RBSDV isolates that were classified as members of group A. The isolates from Sanya (location X) were all SRBSDV isolates that were classified as members of group B.

Comparison of codon usage bias in RBSDV and SRBSDV. RBSDV and SRBSDV S8 sequences are both similarly A+U rich. Codon usage was biased toward codons ending with $\mathrm{A}\left(\mathrm{A} 3_{\mathrm{S}}\right.$ : $42.91 \%)$ or $\mathrm{U}\left(\mathrm{U} 3_{\mathrm{S}}: 49.80 \%\right)$ in RBSDV S8, and codon usage was biased toward codons ending with $\mathrm{A}\left(\mathrm{A} 3_{\mathrm{S}}: 38.04 \%\right)$ or $\mathrm{U}\left(\mathrm{U} 3_{\mathrm{S}}\right.$ : $49.40 \%$ ) in SRBSDV S8. $N c$ and GC values were not significantly different among RBSDV sequences; however these values were significantly higher in SRBSDV than in $\operatorname{RBSDV}(P<0.01)$. $C A I$ values were not significantly different between RBSDV S8 and SRBSDV $\mathrm{S} 8$ but $C A I$ values for $\mathrm{S} 8$ in isolates from locations IV, VI, VII, and $\mathrm{X}$ were significantly higher than those for isolates from location II $(P<0.05)$. In addition, $C B I$ was not significantly different between RBSDV S8 and SRBSDV S8 (Fig. 3A).

The relationships between nucleotide composition and codon bias in RBSDV S8 and SRBSDV S8 are shown as $N c$ plots (a plot of $N c$ versus $\mathrm{GC}_{\mathrm{S}}$ ) in Figure $3 \mathrm{~B}$. If $\mathrm{GC} 3_{\mathrm{S}}$ were the only determinant of $N c$ values, they should fall on a continuous curve between $N c$ and $\mathrm{GC} 3_{\mathrm{S}}$ values. The $N c$ values range from 42 to 47 for RBSDV and from 47 to 50 for SRBSDV,
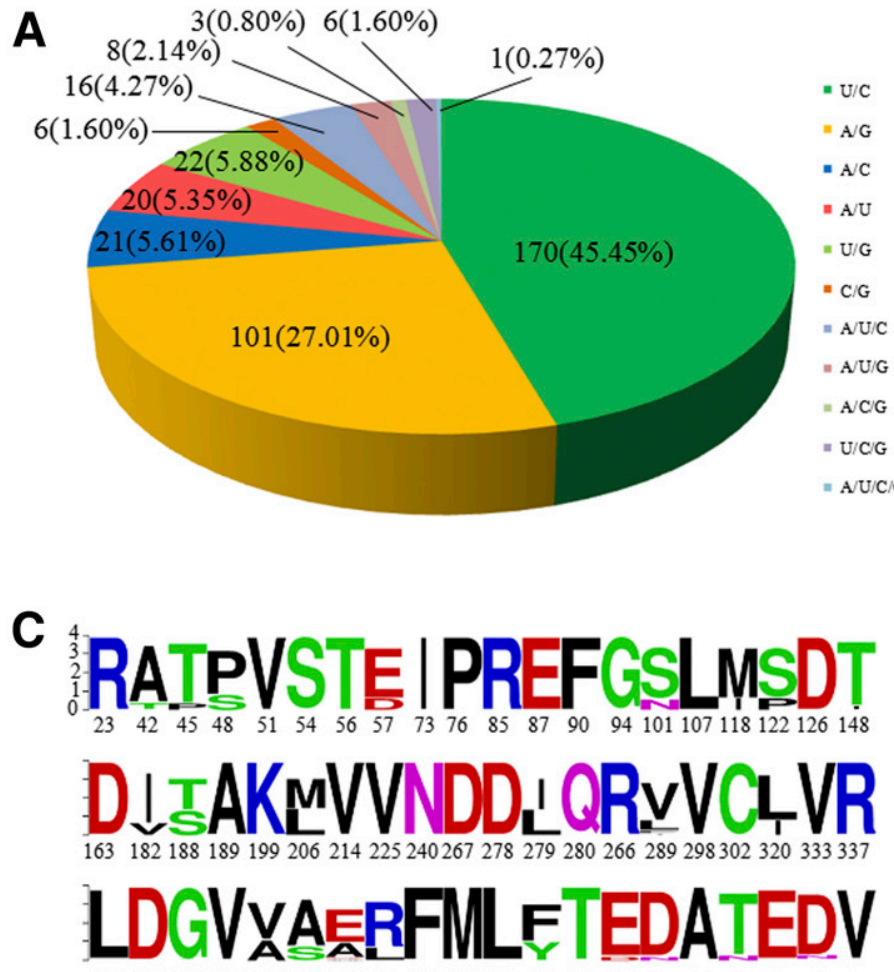

338340355359366369370371386389393401411412421425428429430431 $\mid$ | $A$ |YYSVQESSHYSNFAS| |

445450455463478491497529538540543553556561562575586587589590
B

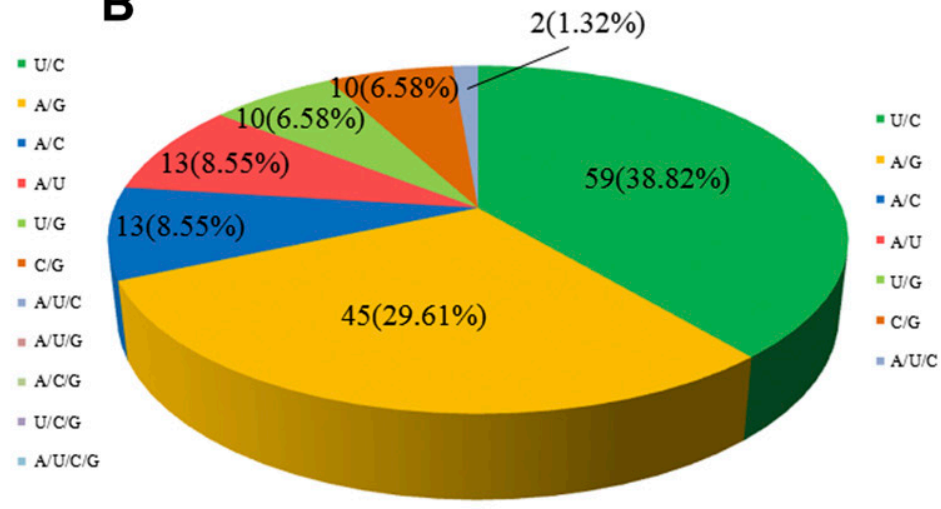

D.INHTYAKSTHNSTKAIDF
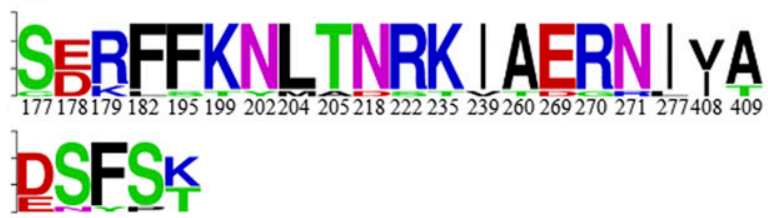

413463469572585

Fig. 4. Nucleotide and amino acid mutations in Rice black-streaked dwarf virus (RBSDV) segment 8 (S8) and Southern rice black-streaked dwarf virus (SRBSDV) S8. A, Mutation analysis of nucleotides in RBSDV S8 sequences. B, Mutation analysis of nucleotides in SRBSDV S8 sequences. C, Amino acid mutation sites in RBSDV S8. D, Amino acid mutation sites in SRBSDV S8. 
which indicates significant differences in codon bias between RBSDV S8 and SRBSDV S8 $(P<0.01)$. All of the points with low $N c$ values lie below the standard curve (Fig. 3C), which suggests additional codon usage bias, independent of $\mathrm{GC}_{\mathrm{S}}$, in RBSDV S8 and SRBSDV S8.

Correspondence analysis of $R S C U$ values for these ORF shows that mutational bias and other parameters have governed the variation in codon usage in S8 in RBSDV and SRBSDV. The first two major axes explain 33.53 and $23.13 \%$ of the variation in RBSDV S8, and 49.04 and $25.93 \%$ of that in SRBSDV S8. The next two axes account for 14.70 and $6.96 \%$ of the variation in RBSDV S8 and 11.33 and $4.41 \%$ of that in SRBSDV S8. Although the first and second axes for RBSDV and SRBSDV S8 data clustered in the plot (Fig. 3D), the majority of data for RBSDV and SRBSDV did not cluster completely. The RBSDV isolates were concentrated mostly in the first quadrant between the two axes, while those of SRBSDV isolates were scattered around the first axis. And although the first axis between RBSDV and SRBSDV was not significant $(P>0.05)$, the second axis between RBSDV and SRBSDV was significant $(P<0.05)$
A

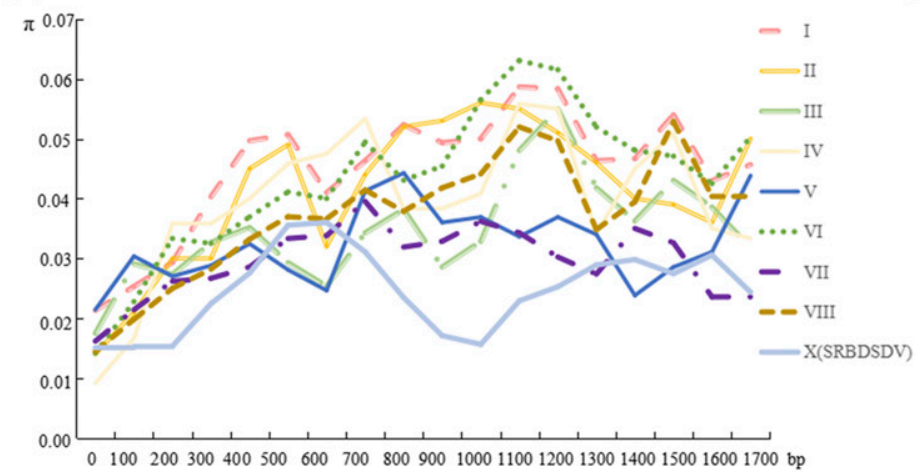

B

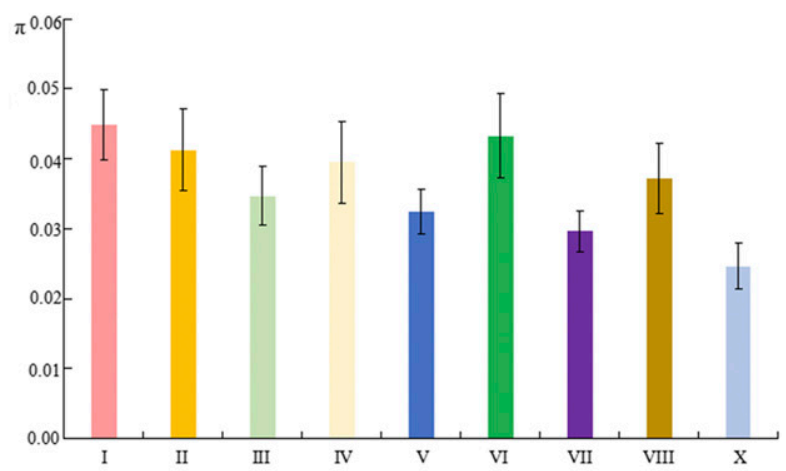

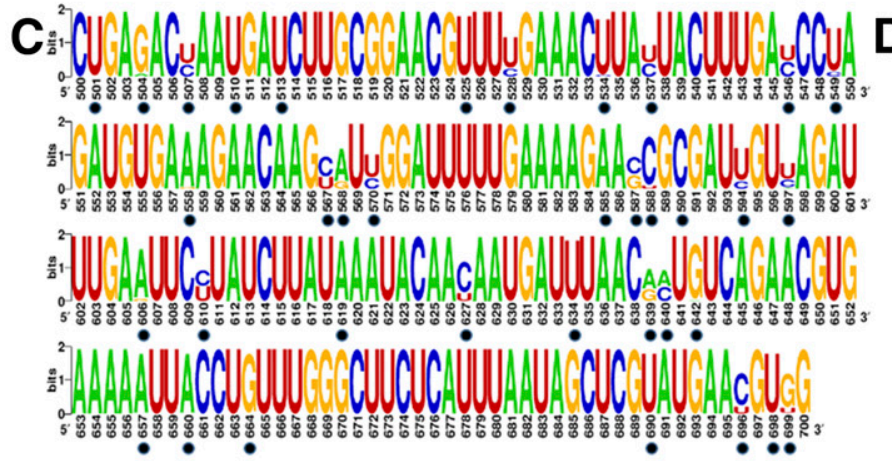

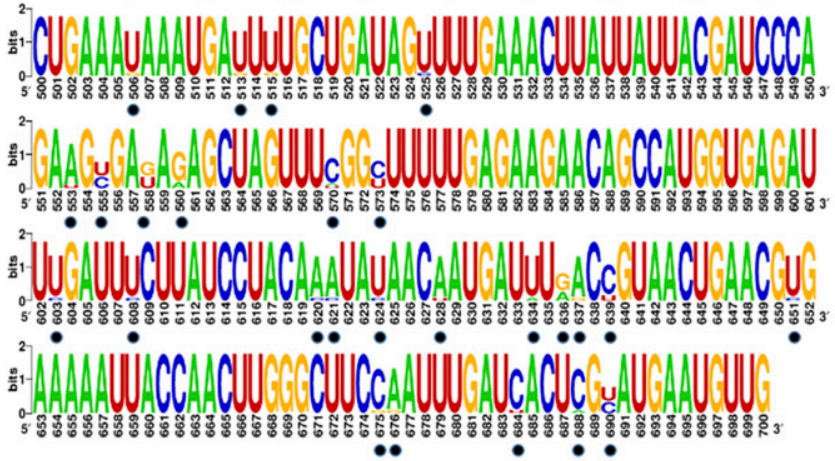

$F$
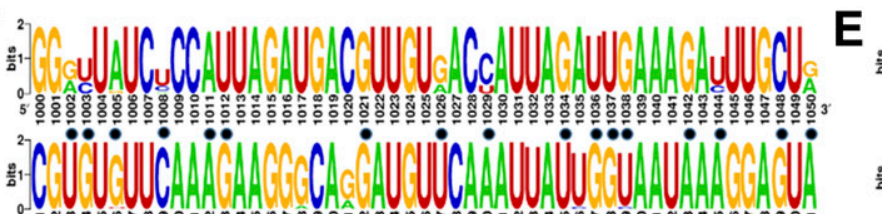
5

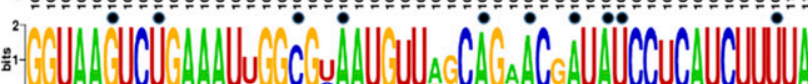
5

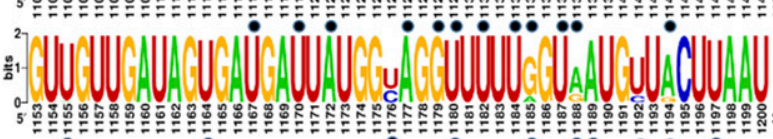

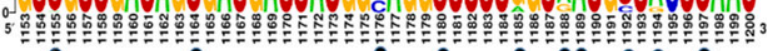

$\mathbf{G}$
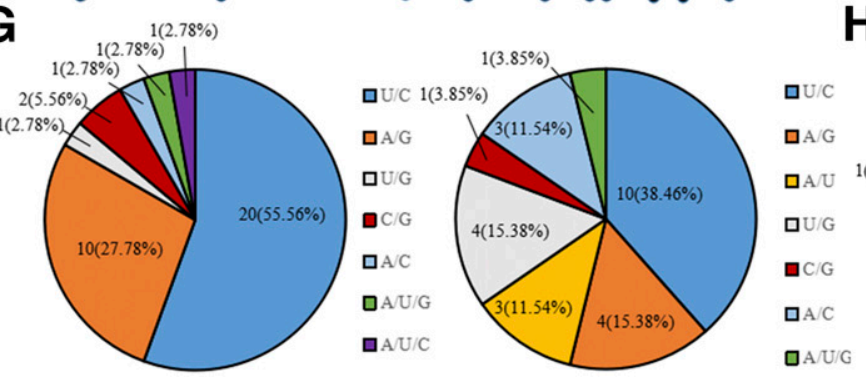

H

${ }^{2}$

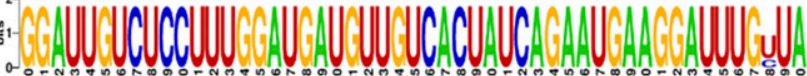

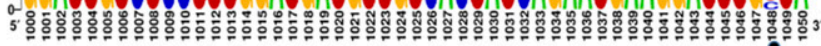

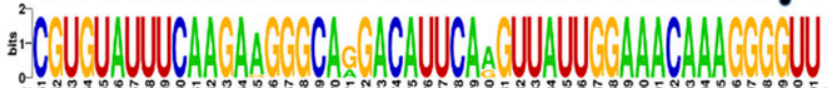
ร.

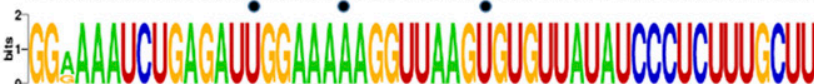
ร.

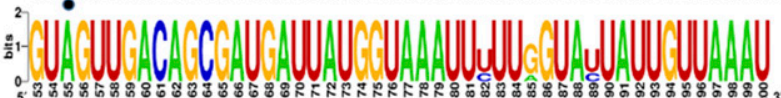

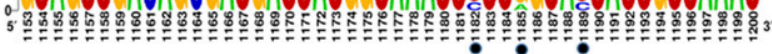
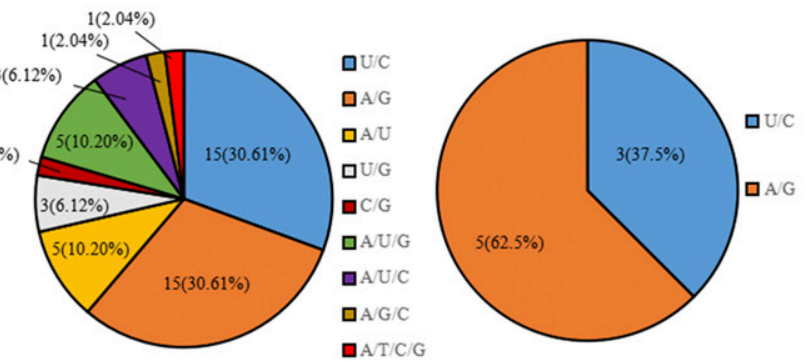

Fig. 5. Nucleotide diversity and variation in segment 8 (S8) from Rice black-streaked dwarf virus (RBSDV) and Southern rice black-streaked dwarf virus (SRBSDV) isolates collected in nine locations in China. Sliding-window analysis of nucleotide diversity in RBSDV S8 in 200-bp sliding windows with 100-bp steps. A, Sliding-window analysis of the nucleotide diversity of S8 between RBSDV and SRBSDV. B, Average nucleotide diversity between RBSDV and SRBSDV. C, Nucleotide mutations in the region from base pairs 500 to 700 in RBSDV S8. D, Nucleotide mutations in the region from base pairs 500 to 700 in SRBSDV S8. E, Nucleotide mutations in the region from base pairs 1,000 to 1,200 in RBSDV S8. F, Nucleotide mutations in the region from base pairs 1,000 to 1,200 in SRBSDV S8. G, Nucleotide mutation analysis in the region from base pairs 500 to 700 in RBSDV S8 and SRBSDV S8. H, Nucleotide mutation analysis in the region from base pairs 1,000 to 1,200 in RBSDV S8 and SRBSDV S8. 
To detect correlations along the first two major axes for both $C A I$ and $N c$, correlation coefficients were calculated among values of these parameters. RBSDV S8 was strongly positively correlated with the $\mathrm{G} 3_{\mathrm{S}}$ value $(r=0.778, P<0.01)$, less strongly correlated with the $C A I$ value $(r=0.577, P<0.01)$, and significantly negatively correlated with the $\mathrm{A}_{3}(r=-0.889, P<0.01)$ and $\mathrm{C} 3_{\mathrm{S}}(r=-0.431$, $P<00.01)$ values on the first axis. RBSDV S8 was correlated with the $C A I$ value $(r=0.465, P<0.01)$ on the second axis. SRBSDV S8 was correlated with the $\mathrm{A} 3_{\mathrm{S}}(r=0.635, P=0.036)$ and $N c(r=0.766$, $P<0.01)$ values on the first axis. SRBSDV S8 was strongly positively correlated with the $\mathrm{T}_{\mathrm{S}}(r=0.821, P<0.01), \mathrm{A}_{\mathrm{S}}(r=$ $0.683, P=0.02)$, and $C A I(r=0.957, P<0.01)$ values and significantly negatively correlated with the G3 S value $(r=-0.980, P<$ $0.01)$ on the second axis. No correlations were detected among the other parameters.

Nucleotide and amino acid sequence variation in RBSDV and SRBSDV. The set of 55 RBSDV and 11 SRBSDV S8 sequences was used to analyze nucleotide variation in these viral genomes and amino acid variation in the proteins they encode. In total, 374 mutations were detected in the RBSDV S8 sequences, which included 138 singleton variable sites and 236 parsimony-informative sites. The single-nucleotide mutations included 271 transitions (U/C: 170; A/G: 101), 69 transversions (A/C: 21; A/U: 20; U/G: 22; C/G: 6), 33 mutations among three possible bases (A/U/C: 16; A/U/G: 8; A/C/G: 3; U/C/G: 6), and only 1 mutation among four possible bases. A 9-bp deletion was detected in the S8 sequences of 13 RBSDV isolates, including 6 isolates from location V, 2 from VI, 2 from VIII, and 1 each from locations I, II, and IX. Four single-nucleotide mutations were detected in the SRBSDV genome within the region corresponding to the 9-bp deletion mutation in RBSDV, and included three U/C mutations and one mutation among three possible bases (A/C/G). In total, 152 mutations were identified in the SRBSDV S8 sequences, including 86 singleton variable sites and 66 parsimonyinformative sites. The 150 single-nucleotide variants included 104 transitions (U/C: 59; A/G: 45), 46 transversions (A/C: 13; A/U: 13; U/G: 10; C/G: 10), and 2 mutations among three possible bases (A/U/C: 2) but no mutations among four possible bases, deletions, or insertions (Fig. 4A).

RBSDV S8 sequences were either 1,927 or 1,936 bp in length, and the 9-bp deletion mutation removed the region from bp 1,807 to 1,815 . The $\mathrm{G}+\mathrm{C}$ content of RBSDV S8 $(34.52 \%)$ was significantly lower than that of SRBSDV $(35.55 \%)(P<0.01)$. The proportion of U/C mutations in RBSDV $(45.45 \%)$ was higher than that in SRBSDV S8 (38.82\%) but the proportions of A/G, A/C, A/U, U/G, and $\mathrm{C} / \mathrm{G}$ mutations $(27.01,5.61,5.35,5.88$, and $1.60 \%$, respectively) were lower in RBSDV than in SRBSDV $(29.61,8.55,8.55,6.58$, and $6.58 \%$, respectively). The proportions of mutations among three possible bases including A/U/C, A/U/G, A/C/G, and U/C/G in RBSDV were $4.27,2.14,0.80$, and $1.60 \%$, respectively. But SRBSDV S8 carried only the A/U/G mutation at $1.32 \%$. One mutation among four possible bases was present in RBSDV S8 but was not found in SRBSDV.
The RBSDV S8 sequence (1,927 bp) was 9 bp shorter than that of RBSDV (1,936 bp) due to the deletion mutation in RBSDV S8 (Fig. 4B).

In total, 80 amino acid changes were detected among the 55 RBSDV P8 sequences, for an average of one substitution site per seven amino acids (Fig. 4C). The deletion mutation in RBSDV was located within the $3^{\prime}$-UTR so it did not affect the amino acid sequences of the P8 protein encoded by S8. In total, 45 variable amino acid sites were detected in SRBSDV, for an average of one substitution site per 13 amino acids (Fig. 4D).

Nucleotide and amino acid sequence diversity of S8. The nucleotide identities of S8 sequences among the 55 RBSDV isolates ranged from 94.1 to $99.9 \%$ and, for the 11 SRBSDV isolates, from 93.9 to $100 \%$. Three SRBSDV S8 nucleotide sequences (15X8, 15X10, and $15 X 11)$ were $100 \%$ identical but no completely identical sequences were found among other RBSDV and SRBSDV nucleotide sequences. The S8 nucleotide sequence identities between RBSDV and SRBSDV isolates ranged from 66.2 to $68.2 \%$, considerably lower than the identities within groups of RBSDV or SRBSDV isolates.

The $\pi$ was calculated across all S8 sequences from the 55 RBSDV and 11 SRBSDV isolates analyzed here. Most polymorphisms in RBSDV S8 were identified in the sequence region spanning from base pairs 1,000 to 1,200 among isolates sampled in location VI. However, most polymorphisms in SRBSDV S8 were identified in the region spanning from base pairs 500 to 700 (Fig. 5A). Although the region from 500 to 700 bp exhibited the most polymorphism in SRBSDV S8, it still exhibited less polymorphism than some isolates of RBSDV, such as those from locations I, II, IV, VI, VII, and VIII (Fig. 5A). In the region from 500 to $700 \mathrm{bp}$, RBSDV contained 36 mutation sites (U/C: 20, A/G: 10, U/G: 1, C/G: 2, A/C: 1, A/U/G: 1, A/U/C: 1) (Fig. 5C) and SRBSDV contained 26 mutations (U/C: 10, A/G: 4, A/U: 3, U/G: 4, C/G: 1, A/C: 3, A/U/G: 1) (Fig. 5D). In the region from 1,000 to 1,200 bp, RBSDV contained 49 mutations (U/C: 15, A/G: 15, A/U: 5, U/G: 3, C/G: 1, A/U/G: 5, A/U/C: 3, A/G/C: 1, A/U/C/G: 1) (Fig. 5E) and SRBSDV contained 8 mutations (U/C: 3, A/G: 5) (Fig. 5F). Mutations U/C and A/G represented the plurality of nucleotide mutations, and all mutation types were more abundant in RBSDV (Fig. $5 \mathrm{G}$ and $\mathrm{H}$ ).

The highest $\pi$ in RBSDV S8 was detected in the isolates from location I $(\pi=0.0449)$, which were significantly more diverse than the sequences from location VII $(\pi=0.0296, P<0.01)$, which were the least diverse. The $\pi$ of RBSDV isolates from location VII was the lowest, and also was not significantly higher than that of the SRBSDV isolates from location $\mathrm{X}(\pi=0.0244, P=0.142)$. Further, the $\pi$ between RBSDV isolates from different regions was significantly higher than that between SRBSDV isolates from different regions (Fig. 5A and B).

Amino acid identity among RBSDV isolates was $98.74 \%$, while that among SRBSDV isolates was $98.71 \%$. Amino acid identity among RBSDV isolates ranged from 95.60 to $100 \%$, while that among SRBSDV isolates ranged from 98.82 to $100 \%$. Amino acid

Table 1. Analysis of recombination between Rice black-streaked dwarf virus (RBSDV) segment 8 (S8) and Southern rice black-streaked dwarf virus (SRBSDV) S8

\begin{tabular}{|c|c|c|c|c|c|c|c|c|c|c|c|}
\hline \multirow[b]{2}{*}{ Recombinant $^{c}$} & \multirow[b]{2}{*}{ Minor parent } & \multirow[b]{2}{*}{ Major parent } & \multicolumn{7}{|c|}{ Detection methods ${ }^{a}$} & \multicolumn{2}{|c|}{ Breakpoint $^{\mathbf{b}}$} \\
\hline & & & $\mathbf{R}$ & G & B & M & $\mathbf{C}$ & $\mathbf{S}$ & $\mathbf{T}$ & Beg & End \\
\hline S8_13IIM-3 & S8_13VM-5 & S8_13IIM-2 & - & + & + & + & + & + & + & 966 & 1,856 \\
\hline S8_13VIM-3 & S8_14IVM-6 & S8_14VIIIR-3 & - & + & + & + & - & + & + & 965 & 1,829 \\
\hline S8_13VIR-5 & S8_14IVM-6 & S8_13IIM-1 & + & + & + & + & + & + & + & 149 & 1,028 \\
\hline S8_13VIIIM-1 & S8_14IVM-6 & S8_13VR-1 & - & + & + & + & + & + & + & 1,047 & 1,865 \\
\hline S8_13VIIIR-2 & S8_13VR-1 & S8_14VIIM-1 & + & + & + & + & + & + & + & 147 & 1,058 \\
\hline S8_14VM-9 & S8_13VM-5 & S8_14IIIM-5 & - & + & + & + & + & + & + & 986 & 1,794 \\
\hline S8_15XM-1 & S8_15XM-3 & S8_15XM-9 & + & + & + & + & - & - & + & 873 & 1,874 \\
\hline S8_15XM-6 & S8_15XM-3 & S8_15XM-9 & - & + & + & + & + & - & + & 855 & 1,873 \\
\hline
\end{tabular}

a Abbreviations R, G, B, M, C, S, and T represent the algorithms in RDP software: RDP, BOOTSCAN, GENECONV, CHIMAERA, Maximum Chi Square, Sister Scanning, and 3Seq, respectively.

${ }^{\mathrm{b}}$ Beginning (Beg) and ending (End) breakpoints.

${ }^{\mathrm{c}}$ Recombinant sequence. 
identities between RBSDV and SRBSDV isolates ranged from 68.53 to $70.90 \%$. These results further demonstrate that the isolates from location X were SRBSDV and those from other locations were RBSDV.

Genetic recombination across the RBSDV and SRBSDV genomes. Analysis of S8 sequences from RBSDV and SRBSDV indicated that recombination events had occurred. Six recombination events were detected in S8 from 55 RBSDV isolates, while two recombination events, 15XM-1 and 15XM-9, were detected in S8 from 11 SRBSDV isolates. Major and minor parental sequences of each recombination event were identified within the sequence of each virus. The major and minor parental sequences reflecting recombination events in RBSDV S8 were identified in isolates from different geographical locations. However, the major and minor parental sequences of two recombination events in SRBSDV S8 were from the same sequences, $15 \mathrm{XM}-3$ and $15 \mathrm{XM}-9$, although the beginning and ending breakpoints of these events differed (Table 1). The recombination events 15XM-1, 15XM-3, and 15XM-9 were located within the deleted sequences from RBSDV. Further, the mutant sequence 13VR-1 that contains the deletion reflected recombination of a major parental sequence with the nonmutated sequence 14IVM-6 from 1,047 to 1,865 bp, which encompassed the deletion.

Tests of selection pressure and neutrality for S8 in RBSDV and SRBSDV. To analyze possible selection pressure on S8 in RBSDV and SRBSDV, the ratios of nonsynonymous to synonymous substitution sites $(\mathrm{Ka} / \mathrm{Ks})$ were calculated for isolates from nine geographic locations. The $K a / K s$ ratios for RBSDV and SRBSDV suggest that these sequences were under negative and purifying selection (Table 2). $K a / K s$ ratios for RBSDV S8 were not significantly lower than those for SRBSDV S8 $(P=0.1550)$. This result suggests that RBSDV and SRBSDV had been subjected to similar levels of selection pressure.

Tajima's D, Fu and Li's D, and Fu and Li's F values for ORF8 were evaluated across the 10 geographical locations studied here. Although Tajima's D, Fu and Li's D, and Fu and Li's F values were not significant $(P>0.05)$, these results showed an expanding trend for RBSDV and SRBSDV populations, except in locations III, IV, VI, or VIII. The haplotype diversity of SRBSDV $(0.9450 \pm 0.0043)$ is lower than that of each RBSDV subpopulation in eight geographic locations (location I: $1 \pm 0.0093$, II: $1 \pm 0.0160$, III: $1 \pm 0.0058$, IV: $1 \pm 0.0313$, V: $1 \pm 0.0020$, VI: $1 \pm 0.0027$, VII: $1 \pm 0.0093$, and VIII: $1 \pm 0.0058$ ) (Table 3).

Analysis of genetic differentiation and gene flow between RBSDV and SRBSDV. Genetic differentiation and gene flow were analyzed for RBSDV and SRBSDV isolates from the 10 geographic locations sampled in this study. The $P$ values for $K s^{*}, \mathrm{Z}$, and $\mathrm{Snn}$ were calculated for RBSDV S8 and SRBSDV S8. These results revealed the significant genetic differentiation of two particular RBSDV S8 subgroups: one comprised isolates from combinations of locations III and $\mathrm{V}$ and the other comprised isolates from combinations of locations V and VII. Gene flow was detected between RBSDV S8 subpopulations

Table 2. Ratio of nonsynonymous to synonymous substitutions in segment 8 sequences from Rice black-streaked dwarf virus (RBSDV) and Southern rice black-streaked dwarf virus (SRBSDV) ${ }^{\mathrm{a}}$

\begin{tabular}{lccc}
\hline Site & $\boldsymbol{K} \boldsymbol{a}$ & $\boldsymbol{K} \boldsymbol{s}$ & $\boldsymbol{K a} / \boldsymbol{K} \boldsymbol{s}$ \\
\hline I & 0.0100 & 0.1861 & 0.05373 \\
II & 0.0085 & 0.1494 & 0.05689 \\
III & 0.0081 & 0.1285 & 0.06304 \\
IV & 0.0074 & 0.1415 & 0.05230 \\
V & 0.0071 & 0.1379 & 0.05149 \\
VI & 0.0096 & 0.1808 & 0.05310 \\
VII & 0.0083 & 0.1342 & 0.06185 \\
VIII & 0.0078 & 0.1552 & 0.05026 \\
X (SRBSDV) & 0.0076 & 0.0924 & 0.08225 \\
RBSDV & 0.0088 & 0.1660 & 0.05301
\end{tabular}

${ }^{\text {a }} \mathrm{Ka}=$ average number of nonsynonymous substitutions per nonsynonymous site, $K s=$ average number of synonymous substitutions per synonymous site, and the $K a / K s$ ratios represent estimated selection pressures. at $F s t<0.33$. Nm values for S8 from RBSDV subpopulations from different geographic locations (except for the combined locations III and $\mathrm{V}$ or V and VII) were $>1$ (Table 4). This result indicates that gene flow between different locations varied. Between some subpopulations, the absolute values of $\mathrm{Nm}$ were $>4$, such as between locations I and II, I and VII, I and VIII, II and IV, II and V, II and VI, III and VII, IV and VII, IV and VIII, VI and VII, and VI and VIII, which showed that gene flow had occurred frequently among these seven subpopulations. The $P$ values of $K s^{*}, Z$, and Snn calculated between the RBSDV populations (I through VIII) and the SRBSDV population (X) of less than 0.01 , together with the values of $N m<1$ and $F s t>0.33$, show that the isolates from location $\mathrm{X}$ were highly genetically differentiated, and that gene flow was not frequent between RBSDV and SRBSDV. This result added further evidence that these two populations are not comprised of the same virus.

\section{Discussion}

SRBSDV is similar to RBSDV in terms of its host range, disease symptoms, serology, and virion morphology (Zhou et al. 2008). Vectored by the SBPH (Wang et al. 2009; Zhou et al. 2011), RBSDV became prevalent in eastern and northern China during the 20th century. Transmitted mainly by the WBPH (Hu 1981; Otuka et al. 2008; Shen et al. 2003), SRBSDV is extensively distributed in southern China and in the Yangtze River basin, south of $29^{\circ} \mathrm{N}$. Some studies have indicated that migration of the viruliferous WBPH might be the main reason that SRBSDV has reached southern China (Cheng et al. 2013).

RBSDV S7 sequences exhibit a low degree of codon usage bias (Zhou et al. 2015b). Calculations in the present study showed that codon usage bias in SRBSDV S8 (average $N c$ of S8 $=47.95$ ) was significantly lower than that in RBSDV S8 (average $N c$ of S8 $=45.01$ ). Codon usage in both RBSDV S8 and SRBSDV S8 are affected by strong mutational bias, which is consistent with previous reports (Adams and Antoniw 2004; Cheng et al. 2012; Jenkins and Holmes 2003; Zhou et al. 2015b). In addition, SRBSDV is more adaptable and exhibits higher gene expression than RBSDV (Cheng et al. 2013). Previous studies have shown that mutation and recombination are two of the main sources of genetic variation in viruses (GarcíaArenal et al. 2003). In the present study, the 9-bp deletion from 1,807 to 1,815 bp was detected in only some RBSDV S8 sequences but was not detected in SRBSDV S8. The corresponding region in SRBSDV contained five mutation sites. The nucleotide identities in S1 through S10 between RBSDV and SRBSDV ranged from 60 to $80 \%$ (Wang et al. 2010; Wang et al. 2003; Zhang et al. 2001a; Zhou et al. 2008). In the present study, the nucleotide identities between RBSDV S8 and SRBSDV S8 ranged from 66.2 to $68.2 \%$, values much lower than those within RBSDV (from 94.1 to $99.9 \%$ ) or SRBSDV (from 93.9 to $100 \%$ ) populations. The $\pi$ values of RBSDV isolates from eight geographical locations (I, II, III, IV, V, VI, VIII, and IX) were significantly higher than those of SRBSDV isolates. However, the RBSDV isolates from location VII exhibited the lowest $\pi$ among RBSDV isolates and did not exhibit significantly higher $\pi$ than SRBSDV isolates. We confirmed results of previous studies, which showed that SRBSDV isolates generally exhibit higher nucleotide

Table 3. Neutrality tests of Rice black-streaked dwarf virus (RBSDV) and Southern rice black-streaked dwarfvirus (SRBSDV) segment 8 subpopulations

\begin{tabular}{lrccc}
\hline Site & Tajima's D & Fu and Li's D & Fu and Li's F & Haplotype diversity \\
\hline I & -0.54727 & -0.65809 & -0.69785 & $1 \pm 0.00926$ \\
II & -0.12696 & -0.1058 & -0.11979 & $1 \pm 0.01600$ \\
III & 0.31568 & 0.39967 & 0.42227 & $1 \pm 0.00583$ \\
IV & 1.76319 & 1.76319 & 1.89024 & $1 \pm 0.03125$ \\
V & -0.72406 & -0.4337 & -0.57262 & $1 \pm 0.00200$ \\
VI & 0.11757 & -0.10261 & -0.05521 & $1 \pm 0.00274$ \\
VII & -0.75133 & -0.6579 & -0.74539 & $1 \pm 0.00926$ \\
VIII & 0.60199 & 0.59951 & 0.66542 & $1 \pm 0.00583$ \\
X & -0.63558 & -0.87115 & -0.92073 & $0.945 \pm 0.00434$ \\
\hline
\end{tabular}

${ }^{a}$ There was no significant difference. 
Table 4. Analysis of genetic differentiation and gene flow between Rice black-streaked dwarf virus and Southern rice black-streaked dwarf virus segment 8 subpopulations ${ }^{\mathrm{a}}$

\begin{tabular}{|c|c|c|c|c|c|c|c|c|}
\hline Subpopulation & $\boldsymbol{K} \boldsymbol{s}^{*}$ & $P$ value & $Z$ & $P$ value & Snn & $P$ value & $F s t$ & $\mathrm{Nm}$ \\
\hline I-II & 4.2054 & 0.5950 & 28.9905 & 0.9070 & 0.5455 & 0.3810 & -0.0553 & -4.77 \\
\hline I-III & 4.0914 & 0.8990 & 39.8825 & 0.7310 & 0.3462 & 0.7480 & -0.0733 & -3.66 \\
\hline I-IV & 4.1072 & 0.8000 & 22.7167 & 0.5770 & 0.3000 & 0.8950 & -0.0923 & -2.96 \\
\hline $\mathrm{I}-\mathrm{V}$ & 4.0080 & 0.0560 & 58.1259 & 0.2430 & 0.6875 & 0.1320 & 0.1126 & 1.97 \\
\hline I-VI & 4.1867 & 0.9300 & 53.1136 & 0.5350 & 0.4556 & 0.6030 & -0.0667 & -4.00 \\
\hline I-VII & 3.9705 & 0.4080 & 33.0000 & 0.5630 & 0.7083 & 0.1290 & -0.0480 & -5.46 \\
\hline I-VIII & 4.1225 & 0.4760 & 38.9577 & 0.4240 & 0.5000 & 0.4670 & -0.0335 & -7.72 \\
\hline II-III & 4.0960 & 0.1050 & 29.0452 & 0.0760 & 0.8750 & 0.0120 & 0.1109 & 2.01 \\
\hline II-IV & 4.1178 & 0.4430 & 18.3800 & 0.5750 & 0.4444 & 0.5660 & -0.0256 & -10.03 \\
\hline II-V & 4.0038 & 0.8310 & 52.8682 & 0.6500 & 0.3778 & 0.8580 & -0.0526 & -5.00 \\
\hline II-VI & 4.1999 & 0.6250 & 48.0911 & 0.9750 & 0.5000 & 0.5590 & -0.0586 & -4.52 \\
\hline II-VII & 3.9585 & 0.1420 & 25.1643 & 0.1440 & 0.7273 & 0.1460 & 0.1021 & 2.20 \\
\hline II-VIII & 4.1311 & 0.6020 & 33.7973 & 0.6500 & 0.5833 & 0.3310 & -0.0711 & -3.77 \\
\hline III-IV & 4.0087 & 0.2780 & 24.5204 & 0.1690 & 0.6212 & 0.4120 & 0.0788 & 2.92 \\
\hline III-V & 3.9559 & $0.0020 * *$ & 56.0095 & $0.0050 * *$ & 0.8235 & $0.0140 *$ & 0.2507 & 0.75 \\
\hline III-VI & 4.1153 & 0.2360 & 53.9582 & 0.0540 & 0.5313 & 0.4170 & 0.0733 & 3.16 \\
\hline III-VII & 3.9593 & 0.2100 & 37.2228 & 0.1800 & 0.7692 & 0.0630 & 0.0256 & 9.52 \\
\hline III-VIII & 4.0433 & 0.0990 & 39.0714 & $0.0250 *$ & 0.7500 & 0.0530 & 0.1228 & 1.79 \\
\hline IV-V & 3.9247 & $0.0330 *$ & 42.7256 & 0.1520 & 0.7857 & 0.0810 & 0.1729 & 1.20 \\
\hline IV-VI & 3.5266 & 0.9840 & 40.8843 & 0.9260 & 0.4231 & 0.8370 & -0.1311 & -2.16 \\
\hline IV-VII & 3.8381 & 0.5020 & 22.1278 & 0.4200 & 0.4333 & 0.6100 & -0.0446 & -5.85 \\
\hline IV-VIII & 4.0363 & 0.4980 & 27.3980 & 0.4470 & 0.4546 & 0.6330 & -0.0442 & -5.91 \\
\hline V-VI & 4.0439 & $0.0470 *$ & 82.2333 & 0.1710 & 0.6842 & 0.0740 & 0.1144 & 1.94 \\
\hline V-VII & 3.8640 & $0.0000 * *$ & 47.4111 & $0.0020 * *$ & 0.8750 & $0.0060 * *$ & 0.3070 & 0.56 \\
\hline V-VIII & 3.9775 & 0.1430 & 65.2117 & 0.1610 & 0.7647 & $0.0340 *$ & 0.0643 & 3.64 \\
\hline VI-VII & 4.0296 & 0.3200 & 50.3503 & 0.1790 & 0.7667 & 0.0660 & 0.0186 & 13.20 \\
\hline VI-VIII & 4.1387 & 0.5920 & 61.5108 & 0.7040 & 0.5313 & 0.3980 & -0.0420 & -6.20 \\
\hline VII-VIII & 3.9305 & 0.1460 & 35.7910 & 0.1340 & 0.6539 & 0.1850 & 0.0901 & 2.52 \\
\hline X-I & 3.7146 & $0.0000 * *$ & 36.3308 & $0.0000 * *$ & 1.0000 & $0.0000 * *$ & 0.8814 & 0.03 \\
\hline X-II & 3.6863 & $0.0010 * *$ & 33.8921 & $0.0010 * *$ & 1.0000 & $0.0010 * *$ & 0.8878 & 0.03 \\
\hline X-III & 3.6897 & $0.0000 * *$ & 38.6940 & $0.0000 * *$ & 1.0000 & $0.0000 * *$ & 0.8929 & 0.03 \\
\hline X-IV & 3.5888 & $0.0000 * *$ & 30.7868 & $0.0000 * *$ & 1.0000 & $0.0000 * *$ & 0.8894 & 0.03 \\
\hline $\mathrm{X}-\mathrm{V}$ & 3.6997 & $0.0000 * *$ & 49.8282 & $0.0000 * *$ & 1.0000 & $0.0000 * *$ & 0.9003 & 0.03 \\
\hline $\mathrm{X}-\mathrm{VI}$ & 3.8032 & $0.0000 * *$ & 45.9599 & $0.0000 * *$ & 1.0000 & $0.0000 * *$ & 0.8844 & 0.03 \\
\hline X-VII & 3.5844 & $0.0000 * *$ & 35.3084 & $0.0010 * *$ & 1.0000 & $0.0010 * *$ & 0.9000 & 0.03 \\
\hline X-VIII & 3.7072 & $0.0000 * *$ & 38.8004 & $0.0000 * *$ & 1.0000 & $0.0000 * *$ & 0.8937 & 0.03 \\
\hline
\end{tabular}

${ }^{a} K s^{*}=$ the sequence statistic, $Z=$ the rank statistic, $\mathrm{Snn}=$ the nearest-neighbor statistic, $F s t=$ among-populations component of genetic variation or normalized variation, and $\mathrm{Nm}=$ product of effective population size and the migration rate among populations. Asterisks: $*$ indicates $0.01<P<0.05$ and $* *$ indicates $0.001<P<0.01$

identity and lower genetic variation than do RBSDV isolates (Cheng et al. 2013).

The RBSDV population in China can be organized into two groups based on the sequences of S1 (Zhou et al. 2016), S7 (Zhou et al. 2015b), S9 (Zhou et al. 2015a), and S10 (Yin et al. 2013), or into three groups based on S8 sequences (Yin et al. 2013), regardless of host, year, or geographic origin. However, in the present study, according to our phylogenetic analysis of S8 sequences, RBSDV and SRBSDV could be divided into two subgroups. Thus, the deletion in S8 likely had an influence on the phylogenetic relationships among these RBSDV and SRBSDV isolates. Recombination events that had occurred in RBSDV and SRBSDV were detected, consistent with previous studies (Li et al. 2012, 2013; Yin et al. 2013). Recombination has been important during the evolution of plant viruses (Roossinck 1997) and nonhomologous recombination could have caused the deletion in RBSDV S8 (Bisaro 1994). Previous studies have shown recombination events to be independent of sample sizes (Zhou et al. 2016) or segment lengths (Zhou et al. 2017). Six recombination events were detected within S8 from 55 RBSDV isolates, while 2 recombination events, $15 \mathrm{XM}-1$ and $15 \mathrm{XM}-9$, were detected in S8 from 11 SRBSDV isolates. However, no recombination events were detected between the S8 sequences of RBSDV and SRBSDV.

Previous studies have shown that RBSDV populations have been under negative and purifying selection (Yin et al. 2013; Zhou et al. 2015 b, 2016), while genetic differentiation and gene flow have mainly been affected by geographical location (Yin et al. 2013). In the present study, although RBSDV and SRBSDV populations showed similar expanding trends, a few isolates from different geographic locations were either stable or contracting. In addition, although RBSDV and SRBSDV were highly similar, RBSDV and SRBSDV were highly genetically differentiated and gene flow was not frequent between these two viruses. Thus, we propose that the deletion mutation in RBSDV S8 was not caused by recombination with SRBSDV S8 and that these viruses might have evolved convergently.

In conclusion, a deletion mutation was detected only in the RBSDV S8 sequence but not in the SRBSDV S8 sequence. The region corresponding to this deletion is prone to mutation and recombination within SRBSDV. RBSDV and SRBSDV could be divided into two subgroups but no recombination was detected between the two viruses. Gene flow between RBSDV and SRBSDV has not been frequent, and there is a high level of genetic differentiation between the two viruses. Although these two viruses are very similar, they can be distinguished by phylogenetic analysis based on nucleotide sequence.

\section{Acknowledgments}

We thank J. Yuan, Z. Meng, S. Tie, Q. Meng, Y. Chen, J. Wu, J. Shi, X. Han, W. Tong, Q. Sun, T. Guo, and Z. Sun for their kind assistance in providing plant samples.

\section{Literature Cited}

Adams, M. J., and Antoniw, J. F. 2004. Codon usage bias amongst plant viruses. Arch. Virol. 149:113-135.

Bisaro, D. M. 1994. Recombination in the Geminiviruses: Mechanisms for Maintaining Genome Size and Generating Genomic Diversity. Springer, Berlin. 
Carver, T. J., and Mullan, L. J. 2005. JAE: Jemboss Alignment Editor. Appl. Bioinf. 4:151-154.

Cheng, X. F., Wu, X. Y., Wang, H. Z., Sun, Y. Q., Qian, Y. S., and Luo, L. 2012. High codon adaptation in citrus tristeza virus to its citrus host. Virol. J. 9:113-121.

Cheng, Z., Li, S., Gao, R., Sun, F., Liu, W., Zhou, G., Wu, J., Zhou, X., and Zhou, Y. 2013. Distribution and genetic diversity of Southern rice black-streaked dwarf virus in China. Virol. J. 10:307-313.

García-Arenal, F., Fraile, A., and Malpica, J. M. 2003. Variation and evolution of plant virus populations. Int. Microbiol. 6:225-232.

Hoang, A. T., Zhang, H., Yang, J., Chen, J., Hébrard, E., Zhou, G., Vinh, V. N., and Cheng, J. 2011. Identification, characterization, and distribution of Southern rice black-streaked dwarf virus in Vietnam. Plant Dis. 95:1063-1069.

Hu, G. 1981. Research progress in migration of rice whitebacked planthopper. Sci. Agric. Sin. 10:25-30

Hudson, R. R. 2000. A new statistic for detecting genetic differentiation. Genetics 155:2011-2014.

Hudson, R. R., Boos, D. D., and Kaplan, N. L. 1992. A statistical test for detecting geographic subdivision. Mol. Biol. Evol. 9:138-151.

Isogai, M., Uyeda, I., and Lee, B. C. 1998. Detection and assignment of proteins encoded by rice black streaked dwarf fijivirus S7, S8, S9 and S10. J. Gen. Virol. 79:1487-1494.

Jenkins, G. M., and Holmes, E. C. 2003. The extent of codon usage bias in human RNA viruses and its evolutionary origin. Virus Res. 92:1-7.

Li, Y. Q., Jia, M. G., Jiang, Z. D., Zhou, T., and Fan, Z. F. 2012. Molecular variation and recombination in RNA segment 10 of rice black-streaked dwarf virus isolated from China during 2007-2010. Arch. Virol. 157:1351-1356.

Li, Y. Q., Xia, Z. H., Peng, J., Zhou, T., and Fan, Z. F. 2013. Evidence of recombination and genetic diversity in Southern rice black-streaked dwarf virus. Arch. Virol. 158:2147-2151.

Librado, P., and Rozas, J. 2009. DnaSP v5: A software for comprehensive analysis of DNA polymorphism data. Bioinformatics 25:1451-1452.

Liu, H., Ni, Y. Q., Rao, L. X., Wu, J. X., and Zhou, X. P. 2013. Development of monoclonal antibodies against Southern rice black-streaked dwarf virus and Rice black-streaked dwarf virus and their application in virus detection. Acta Phytopathol. Sin. 43:27-34

Liu, H. J., Wei, C. H., Zhong, Y. W., and Li, Y. 2007. Rice black-streaked dwarf virus minor core protein P8 is a nuclear dimeric protein and represses transcription in tobacco protoplasts. FEBS Lett. 581:2534-2540.

Martin, D. P., Lemey, P., Lott, M., Moulton, V., Posada, D., and Lefeuvre, P. 2010. RDP3: A flexible and fast computer program for analyzing recombination. Bioinformatics 26:2462-2463.

Marzachí, C., Boccardo, G., and Nuss, D. L. 1991. Cloning of the maize rough dwarf virus genome: Molecular confirmation of the plant-reovirus classification scheme and identification of two large nonoverlapping coding domains within a single genomic segment. Virology 180:518-526.

Morris, T. J., and Dodds, J. A. 1979. Isolation and analysis of double-stranded RNA from virus-infected plant and fungal tissue. Phytopathology 69:854-858.

Otuka, A., Matsumura, M., Watanabe, T., and Dinh, T. V. 2008. A migration analysis for rice planthoppers, Sogatella furcifera (Horváth) and Nilaparvata lugens (Stål) (Homoptera: Delphacidae), emigrating from northern Vietnam from April to May. Appl. Entomol. Zool. (Jpn.) 43:527-534.

Pamilo, P., and Bianchi, N. O. 1993. Evolution of the Zfx and Zfy genes: Rates and interdependence between the genes. Mol. Biol. Evol. 10:271-281.

Roossinck, M. J. 1997. Mechanisms of plant virus evolution. Annu. Rev. Phytopathol. 35:191-209.

Rozas, J. 2009. DNA sequence polymorphism analysis using DnaSP. Methods Mol Biol. 537:337-350.

Sau, K., Gupta, S. K., Sau, S., Mandal, S. C., and Ghosh, T. C. 2006. Factors influencing synonymous codon and amino acid usage biases in Mimivirus. Biosystems 85:107-113.

Shackelton, L. A., Parrish, C. R., and Holmes, E. C. 2006. Evolutionary Basis of Codon Usage and Nucleotide Composition Bias in Vertebrate DNA Viruses. J. Mol. Evol. 62:551-563.

Sharp, P. M., and Li, W. H. 1987. The codon adaptation index-a measure of directional synonymous codon usage bias, and its potential applications. Nucleic Acids Res. 15:1281-1295

Shen, J. H., Shang, J. M., and Liu, G. J. 2003. Management of the white-backed planthopper, Sogatella furcifera in China: A mini-review. Chin. J. Rice Sci. 17:7-22.

Shikata, E., and Kitagawa, Y. 1977. Rice black-streaked dwarf virus: Its properties, morphology and intracellular localization. Virology 77:826-842.
Tajima, F. 1989. Statistical method for testing the neutral mutation hypothesis by DNA polymorphism. Genetics 123:585-595.

Tamura, K., Stecher, G., Peterson, D., Filipski, A., and Kumar, S. 2013. MEGA6: Molecular Evolutionary Genetics Analysis version 6.0. Mol. Biol. Evol. 30: 2725-2729.

Wang, H. D., Chen, J. P., Wang, A. G., Jiang, X. H., and Adams, M. J. 2009. Studies on the epidemiology and yield losses from rice black-streaked dwarf disease in a recent epidemic in Zhejiang province, China. Plant Pathol. 58 815-825.

Wang, Q., Jian, Y., Zhou, G.-H., Zhang, H.-M., Chen, J.-P., and Adams, M. J. 2010. The complete genome sequence of two isolates of Southern rice black streaked dwarf virus, a new member of the genus Fijivirus. J. Phytopathol. 158:733-737.

Wang, Z., Yu, D., Li, X., Zeng, M., Chen, Z., Bi, L., Liu, J., Jin, L., Hu, D., and Yang, S. 2012. The development and application of a Dot-ELISA assay for diagnosis of southern rice black-streaked dwarf disease in the field. Viruses 4:167-183.

Wang, Z.-H., Fang, S.-G., Xu, J.-L., Sun, L.-Y., Li, D.-W., and Yu, J.-L. 2003 Sequence analysis of the complete genome of rice black-streaked dwarf virus isolated from maize with rough dwarf disease. Virus Genes 27:163-168.

Weng, J. F., Li, B., Liu, C. L., Yang, X. Y., Wang, H. W., Hao, Z. F., Li, M. S. Zhang, D. G., Ci, X. K., Li, X. H., and Zhang, S. H. 2013. A nonsynonymous SNP within the isopentenyl transferase 2 locus is associated with kernel weight in Chinese maize inbreds (Zea mays L.). BMC Plant Biol. 13:98-108.

Wright, F. 1990. The 'effective number of codons' used in a gene. Gene 87 23-29.

Wright, S. 1951. The genetical structure of populations. Ann Eugenics. 15: 323-354.

Yin, X., Xu, F. F., Zheng, F. Q., Li, X. D., Liu, B. S., and Zhang, C. Q. 2011 Molecular characterization of segments S7 to S10 of a Southern rice blackstreaked dwarf virus isolate from maize in northern China. Virol. Sin. 26:47-53.

Yin, X., Zheng, F. Q., Tang, W., Zhu, Q. Q., Li, X. D., Zhang, G. M., Liu, H. T. and Liu, B. S. 2013. Genetic structure of rice black-streaked dwarf virus populations in China. Arch. Virol. 158:2505-2515.

Zhang, H. M., Chen, J. P., and Adams, M. J. 2001a. Molecular characterisation of segments 1 to 6 of Rice black-streaked dwarf virus from China provides the complete genome. Arch. Virol. 146:2331-2339.

Zhang, H. M., Chen, J. P., Lei, J. L., and Adams, M. J. 2001b. Sequence analysis shows that a dwarfing disease on rice, wheat and maize in China is caused by rice black-streaked dwarf virus. Eur. J. Plant Pathol. 107:563-567.

Zhang, H. M., Yang, J., Chen, J. P., and Adams, M. J. 2008. A black-streaked dwarf disease on rice in China is caused by a novel Fijivirus. Arch. Virol. 153:1893-1898.

Zhou, G. H., Wen, J. J., Cai, D. J., Li, P., Xu, D. L., and Zhang, S. G. 2008 Southern rice black-streaked dwarf virus: A new proposed Fijivirus species in the family Reoviridae. Sci. Bull. (Beijing) 53:3677-3685.

Zhou, G. H., Xu, D. L., Xu, D. G., and Zhang, M. X. 2013. Southern rice blackstreaked dwarf virus: A white-backed planthopper-transmitted Fijivirus threatening rice production in Asia. Front. Microbiol. 4:270.

Zhou, T., Wu, L. J., Wang, Y., Cheng, Z. B., Ji, Y. H., Fan, Y. J., and Zhou, Y. J. 2011. Transmission of Rice black-streaked dwarf virus from frozen infected leaves to healthy rice plants by small brown planthopper (Laodelphax striatellus). Rice Sci. 18:152-156.

Zhou, Y., Meng, Q. C., Chen, Y. P., Wu, J. R., Hao, Z. F., Wang, Z. H., Zhang, D. G., Li, M. S., Yong, H. J., Zhang, S. H., Li, X. H., and Weng, J. F. 2016. Molecular variation and expansion of a rice black-streaked dwarf virus population based on analysis of segment 1 in Jining, China. Arch. Virol. 161: 3435-3443

Zhou, Y., Weng, J. F., Chen, Y. P., Liu, C. L., Han, X. H., Hao, Z. F., Li, M. S., Yong, H. J., Zhang, D. G., Zhang, S. H., and Li, X. H. 2015a. Phylogenetic and recombination analysis of rice black-streaked dwarf virus segment 9 in China. Arch. Virol. 160:1119-1123.

Zhou, Y., Weng, J. F., Chen, Y. P., Wu, J. R., Meng, Q. C., Han, X. H., Hao, Z. F., Li, M. S., Yong, H. J., Zhang, D. G., Zhang, S. H., and Li, X. H. 2015b. Molecular genetic analysis and evolution of segment 7 in Rice black-streaked dwarf virus in China. PLoS One 10:e0131410.

Zhou, Y., Zhang, L., Zhang, X. M., Zu, H. Y., Di, H., Dong, L., Liu, X. J., Zeng, X., Weng, J. F., Wang, Z. H., and Li, X. H. 2017. Rice black-streaked dwarf virus genome in China: Diversification, phylogeny and selection. Plant Dis. 101:1588-1596. 